\title{
Effect of goshajinkigan, a Japanese herbal medicine, on the plasma neuropeptides in humans
}

\author{
Yuhki Sato*, Ryosuke Tatsuta, Yosuke Suzuki and Hiroki Itoh \\ Department of Clinical Pharmacy, Oita University Hospital, 1-1 Idaigaoka, Hasama-machi, Oita 879-5593, Japan
}

\begin{abstract}
A traditional Japanese Kampo medicine goshajinkigan has been empirically used for the treatment of numbness, cold sensation and limb pain associated with diabetic neuropathy especially. One of the mechanisms of the empirical effects is assumed to be due to local changes in neuropeptide levels. The purpose of this study is to examine the effect of gosyajinkigan on calcitonin gene-related peptide (CGRP)-, substance $\mathrm{P}$-, and vasoactive intestinal polypeptides (VIP)-like immunoreactive substances (IS) in plasma taken from healthy humans. Gosyajinkigan $(4.5 \mathrm{~g})$ or placebo was orally administered to seven healthy males. Blood samples were taken before, and at 20, 40,60,90,120,180, and 240 min after administration, followed by the extracting procedure, and submitted to a highly sensitive enzyme immunoassay system for CGRP-, substance P-, and VIP-IS. A single oral administration of goshajinkigan caused significant increases in plasma CGRP-, and VIPIS levels compared with placebo. In this study, we concluded that goshajinkigan might improve peripheral nervous dysfunction via neuropeptide release as CGRP and VIP.
\end{abstract}

Abbreviations: CGRP: Calcitonin Gene-related Peptide; VIP: Vasoactive Intestinal Polypeptide; EIA: Enzyme Immunoassay

\section{Introduction}

Gastrointestinal motility is mainly regulated by two factors including humoral hormones and nervous transmitters from both central and peripheral nervous system. Neuroeptides exert action on gastrointestinal motility via both endocrine hormones and peptidergic transmitters [1]. These peptides as modulatory mediators appear to be major components of bodily integration and have important regulatory actions on physiological function of gastrointestinal tract. Recent studies have indicated that some disorders of gastrointestinal motility following surgery and chronic stresses are related to neuropeptides, such as vasoactive intestinal peptide (VIP), calcitonin gene-related peptide (CGRP), and substance P (Figure 1) [2,3].

Traditional herbal medicines have been employed for a few thousand years and have contributed greatly to the treatment of many subjective symptoms. Goshajinkigan is a Kampo medicine (traditional Japanese medicine) consisting of ten crude drugs, this formula is traditionally used for improvement of symptoms like numbness, cold sensation and limb pain associated with diabetic neuropathy [4]. Moreover, it has been reported that peripheral neurotoxicity due to

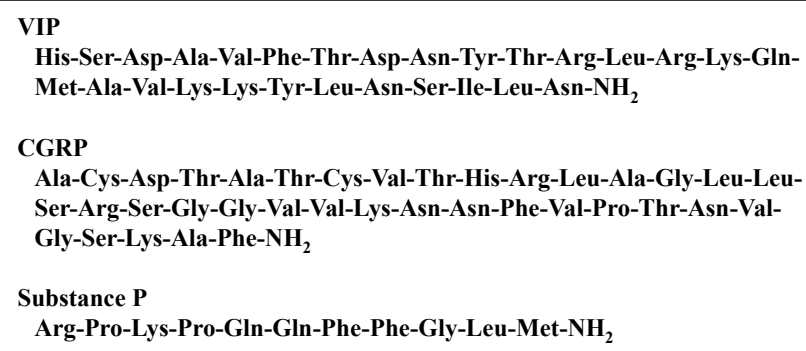

Figure 1. Primary structures of neuropeptides. anticancer drugs such as oxaliplatin was relieved by administration of goshajinkigan in patients with advanced colorectal cancer [5]. It has been reported that goshajinkigan has been especially evaluated for its clinical usefulness in the treatment of pain control in lower extremities, numbness, blurred vision, difficulty in urination, and edema [5]. There are some reports that Kampo medicines which include aconiti tuber have been used to improve the health of persons with a weak constitution and metabolism.And the pharmacological effects of Aconiti tuber have been described as positive inotropic effects [6].

In recent years, some Kampo medicines have been elucidated pharmacologically from the viewpoint of neuropeptide levels [7-10]. Among the medicines, daikenchuto potentially enhance gastrointestinal motility, similar to the gastrointestinal prokinetic drugs like cisapride and metoclopramide [11], and raised plasma neuropeptide levels in healthy humans and patients with morphine-induced constipation [7-10]. These results indicate new pharmacological effects of this drug. At present, there are also few reports that have examined the temporal relationship among the neuropeptides and goshajinkigan. Thus, we hypothesized that goshajinkigan could induce alterations of neuropeptides in plasma, which could be involved in the pathogenesis of the nervous dysfunction. The purpose of this study was to determine whether clinical effects of goshajinkigan are due to changes in the plasma CGRP-, substance P-, and VIP-like immunoreactive substances (IS) levels after the administration of goshajinkigan.

Correspondence to: Yuhki Sato, Department of Clinical Pharmacy, Oita University Hospital, 1-1 Idaigaoka, Hasama-machi, Oita 879-5593, Japan, Tel: +81-97-586-6112; Fax: +81-97-586-6119; E-mail: syuhki@ oita-u.ac.jp

Key words: Japanese kampo medicine, goshajinkigan, neuropeitide, enzyme immunoassay

Received: June 08, 2015; Accepted: July 25, 2015; Published: August 01, 2015 


\section{Materials and methods}

\section{Materials}

Goshajinkigan was purchased from Tsumura Co. Ltd. (Tokyo, Japan) Ratio of respective crude drug component was as follows: Rehmanniae radix $(5.0 \mathrm{~g})$, Achyranthis radix (3.0 g), Corni fructus $(3.0 \mathrm{~g})$, Moutan cortex (3.0 g), Alismatics rhizome (3.0 g), Dioscorea rhizome (3.0 g), Plantaginis semen (3.0 g), Hoelen (3.0 g), processed Aconiti tuber $(1.0 \mathrm{~g})$, and Cinnamomi cortex $(1.0 \mathrm{~g})$. The dosage of goshajinkigan was determined based on maximum daily dose used in clinical therapy. The placebo was crystalline cellulose and lactose, in the ratio of 1:1. Synthetic human VIP, CGRP and its fragment (8-37), and substance $P$, were purchased from the Peptide Institute (Osaka, Japan). The VIP fragment peptide was supplied by Professor $\mathrm{H}$. Yajima (Kyoto University, Kyoto, Japan). Antisera to VIP (A604/R1B) and CGRP (CA1132) were purchased from Biogenesis (Poole, UK), and substance P (RA-08-095) from Cambridge Research Biochemicals (Cambridge, UK). Goat affinity-purified antibody to rabbit IgG (whole molecule) (55641) was purchased from ICN Pharmaceuticals (Aurora, $\mathrm{OH}, \mathrm{USA}$ ). All other reagents were of analytical reagent grade from commercial sources.

\section{Subjects}

Seven healthy male volunteers (nonsmokers), aged 24-35 years (median 27 years), weighing 53-72 kg (median weight $60 \mathrm{~kg}$ ), participated in this study. Each subjects received information about the study's scientific purpose, which was approved by the Ethics Committee of Oita Medical University, and gave informed consent. No subject had received any medication for 1 month preceding the test and no stimulator of gastrointestinal motility was administered to any subjects during the study.

\section{Study schedule}

Goshajinkigan (7.5 g) or placebo was orally administered with 100 $\mathrm{mL}$ water. All subjects followed the same drug-placebo sequence. Each subjects was administered these drugs with an interval of four weeks. Venous blood samples $(10 \mathrm{~mL})$ from a forearm vein just before the drug was administered, at 20, 40,60, 90, 120, 180, and $240 \mathrm{~min}$ after administration of the test substances. In addition, it is reported that plasma CGRP of from 14:00 to 18:00, substance P and the VIP density do not have the physiological change $[12,13]$. The study was carried out from 14:00 to 18:00.

\section{Preparation of plasma extracts}

Thebloodsampleswerecollectedinachilled tubecontainingaprotinin (500-kallikrein inhibitor units $/ \mathrm{mL}$ ) and ethylenediaminetetraacetic acid (EDTA) $(1.2 \mathrm{mg} / \mathrm{mL})$. After centrifugation, the plasma samples were diluted with $4 \%$ acetic acid ( $\mathrm{pH} 4.0$ ) and loaded onto Sep-Pak $\mathrm{C}_{18}$ cartridges (Millipore Corp., Milford, USA), and washed with $4 \%$ acetic acid. The peptides in the plasma were eluted with $70 \%$ acetonitrile in $0.5 \%$ acetic acid ( $\mathrm{pH} 4.0)$, lyophilized, and reconstituted to $100 \mu \mathrm{L}$ with the assay buffer and subjected to EIA. For the EIA system, plasma samples were concentrated five-fold with Sep-Pak $\mathrm{C}_{18}$ cartridges. The recovery of plasma CGRP-, substance P-, and VIP-IS was $>90 \%$ with this extraction procedure.

EIAs of, CGRP, substance P, and VIP EIAs for CGRP- [12], substance P- [14], and VIP-IS [15] were performed as previously described, by a delayed-addition method [16]. Separation of bound and free antigen was performed on an anti-rabbit IgG coated immunoplates.
The fluorescent product 4-methylumbelliferon was measured with an MTP-100F microplate reader (Corona Electric, Ibaraki, Japan). Human fragment VIP (11-28), human CGRP (8-37) and substance P were conjugated with $\beta$-D-galactosidase (Boehringer Mannheim, Mannheim, Germany) with N-( $\varepsilon$-maleimidocaproyloxy)-succimide according to the method of Kitagawa et al. [17]. The EIAs for VIP, CGRP, and substance $P$ was specific and highly sensitive to detection limits of $1.00,0.08$ and $0.40 \mathrm{fmol} /$ well, respectively.

\section{Statistical analysis}

All values are expressed as the mean \pm s.d. The statistical significance evaluated by analysis of variance for repeated measure followed by the Dunnet' test. Value of $\mathrm{p}<0.01$ or $\mathrm{p}<0.05$ was considered to represent a statistically significant difference.

\section{Results and discussion}

Goshajinkigan has been used for thousands of years to improve gastrointestinal functions. It has been reported that goshajinkigan significantly improves the peripheral neuropathy. Previous studies have shown that the effects of peripheral blood flow are mainly regulated by hormonal and neuronal mechanisms, which may be related to neuropeptides. Therefore, in this study, CGRP, substance P, and VIP, which regulate peripheral blood flow, were examined to study the effects of goshajinkigan.

The plasma CGRP- and substance P-IS level-time profile after administration of goshajinkigan to healthy subjects is summarized in Figures 2 and 3. Goshajinkigan significantly increased CGRP-IS at 60 $\min (15.5 \pm 2.4 \mathrm{pg} / \mathrm{mL})$ compared with the response of the placebo group $(7.5 \pm 0.9 \mathrm{pg} / \mathrm{mL})$. But Goshajinkigan did not, however, alter levels of substance P.

The plasma VIP-IS level-time profile after administration of goshajinkigan to healthy subjects are shown in Figure 3. Goshajinkigan significantly increased VIP-IS at 30, 60 and $120 \mathrm{~min}(5.7 \pm 0.4 \mathrm{pg} / \mathrm{mL}$ at $30 \mathrm{~min}, 5.5 \pm 0.6 \mathrm{pg} / \mathrm{mL}$ at $60 \mathrm{~min}$ and $5.0 \pm 1.1 \mathrm{pg} / \mathrm{mL}$ at $120 \mathrm{~min}$ ) compared with placebo $(4.0 \pm 1.0 \mathrm{pg} / \mathrm{mL}$ at $30 \mathrm{~min}, 3.7 \pm 0.6 \mathrm{pg} / \mathrm{mL}$ at $60 \mathrm{~min}$ and $3.5 \pm 0.7 \mathrm{pg} / \mathrm{mL}$ at $120 \mathrm{~min})$.

CGRP has several potent biological activities, including vasodilation, and in the gastrointestinal mucosa its vasodilatory effects following stimulated release from the extrinsic sensory innervation is considered to serve as an important protective mechanism for

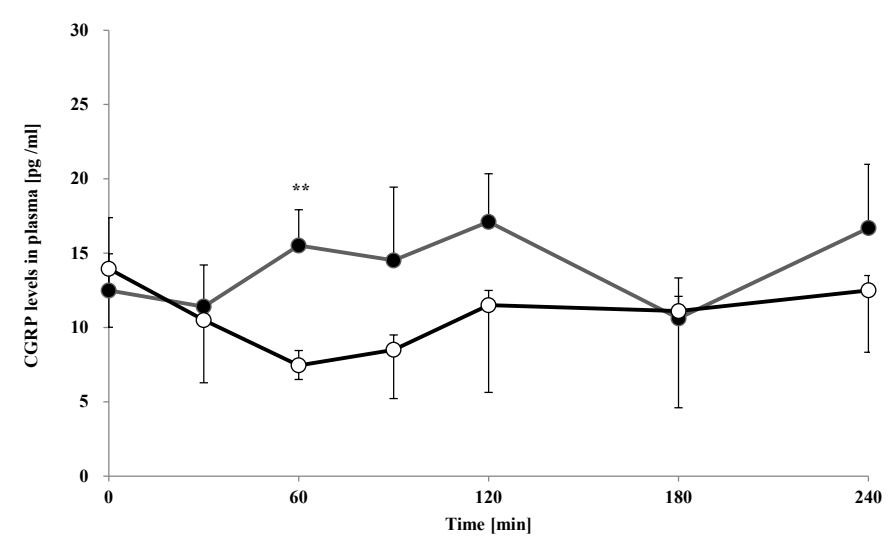

Figure 2. Changes of oral goshajinkigan $7.5 \mathrm{~g}(\bullet)$ or placebo (०) on plasma CGRP-IS levels in healthy subjects. Each point represents the mean \pm S.D., $\mathrm{n}=7$. $* * \mathrm{P}<0.01$ compared with placebo 


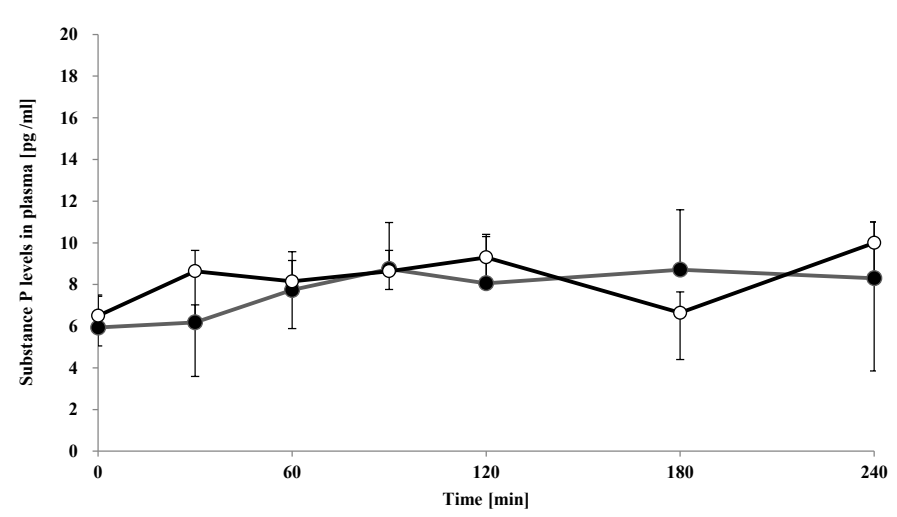

Figure 3. Effect of oral goshajinkigan $7.5 \mathrm{~g}(\bullet)$ or placebo (०) on plasma substance P-IS levels in healthy subjects.

Each point represents the mean \pm S.D., $\mathrm{n}=7$.

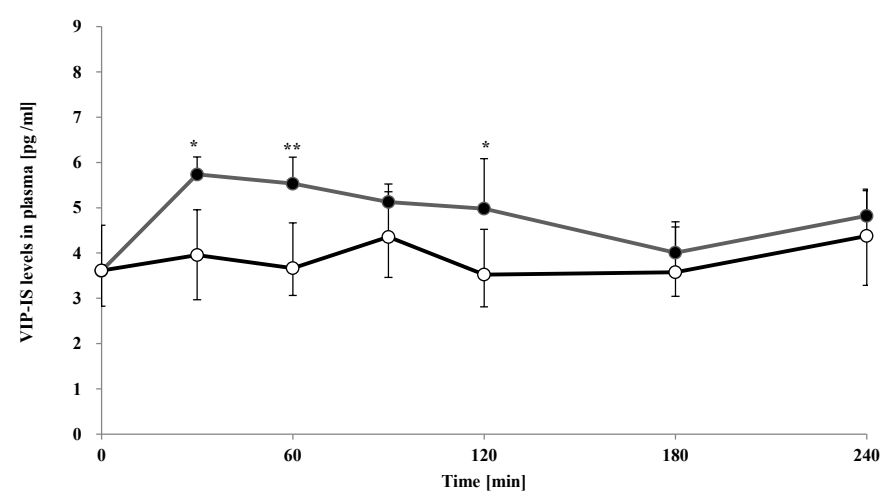

Figure 4. Effect of oral goshajinkigan $7.5 \mathrm{~g}(\bullet)$ or placebo (०) on plasma VIP-IS levels in healthy subjects. Each point represents the mean \pm S.D., $\mathrm{n}=7$. $* * \mathrm{P}<0.01$ and $* \mathrm{P}<0.05$ compared with placebo.

maintaining mucosal integrity [18]. In addition, CGRP is also present in enteric neurons [19] and has a potent effect on gastrointestinal motility and secretion [20]. Previous studies have reported that the intrinsic sensory pathway, which mediates the peristaltic response to mucosal stimulation, utilizes CGRP as a sensory transmitter [21]. Furthermore, CGRP is a potent intestinal vasodilator in conscious dogs and causes increases in the intestinal blood flow [22]. CGRP in blood mainly comes from the gut and vessels. CGRP may be released extensively from the nerve endings around vessels into circulation through organ stimulation such as stress and intestinal ischemia-reperfusion injury in the rat. In this study, goshajinkigan significantly raised plasma CGRPIS levels. Accordingly, the mechanisms of goshajinkigan, based on the increment of CGRP-IS levels in human plasma, may include the increments in intestinal and peripheral blood flow, which is mediated by CGRP. Gosyajinkigan prevents oxaliplatin-induced acute peripheral neuropathy by suppression of transient receptor potential vanilloid 1 (TRPV1) at the spinal cord. Substance P and CGRP regulated by TRPV1 at spinal level contribute to oxaliplatin-induced neuropathic pain [23]. It might be thought that goshajinkigan prevents peripheral neurotoxicity by chemotherapy via CGRP.

VIP is widely distributed in the central and peripheral nervous system. This peptide has a vasodilating effect and increase peripheral blood flow. VIP is also known as a major regulator of mammalian intestinal motility and induces relaxation of precontracted ileal longitudinal muscle, and mediates the peristalitic reflex [24]. In our results, goshajinkigan increased plasma VIP-IS levels. This effect might be due to Aconiti Tube that contains in goshajinkigan. Preparations of Aconiti Tuber have been therapeutically used to increase peripheral body temperature. This herb contains aconite alkaloids (aconitine, and mesaconitine etc.) as its bioactive components. In a previous study, it was reported that mesaconitine elicited a strong relaxation in isolated rat aorta, and this relaxation is manly endothelium-dependent and mediated by nitric oxide (NO), a powerful vasodilator agent [25]. Suzuki et al reported that goshajinkigan has been shown to increase peripheral blood flow and exhibit analgesic effects by increasing NO production [26]. It is known that $\mathrm{NO}$ is a neuronal co-mediator of $\mathrm{VIP} /$ cholinergic vasodilation, and colocalized with VIP in myenteric plexus [27]. Although it has not been demonstrated previously whether Aconiti tuber directly stimulates VIP-containing nerves, this herb or others combinations might be closely related to the release of VIP in autonomic nervous system.

In this study, the vital signs such as blood pressure (diastolic and systolic pressure) and the pulse in had not changed after administration of goshajinkigan or placebo (data not shown).

We concluded that goshajinkigan might improve peripheral nervous dysfunction by significantly increasing CGRP-, and VIP-IS levels in plasma. However, as the present results for the number of cases is small, it is difficult to simply compare the effect thus, further consideration is required in the future. The effects of goshajinkigan on neuropeptides were found in healthy subjects, so essentially, the effects of goshajinkigan are necessary to investigate the neuropeptide levels on the pathological condition and at local site.

\section{Conflicts of Interest}

The authors state that they have no conflicts of interests related to this study.

\section{Acknowledgments}

The authors thank all members of pharmacy at Oita University Hospital for assistance with data collection.

\section{Ethical approval}

The present study was carried out in accordance with the guidelines for the care for human studies adopted by the Ethics Committee of Oita University of Medicine, and notified by the Japanese government.

\section{References}

1. Rogers RC, McTigue DM, Hermann GE (1996) Vagal control of digestion: modulation by central neural and peripheral endocrine factors. Neurosci Biobehav Rev 20: 57-66. [Crossref]

2. Zittel TT, Lloyd KC, Rothenhöfer I, Wong H, Walsh JH, et al. (1998) Calcitonin generelated peptide and spinal afferents partly mediate postoperative colonic ileus in the rat. Surgery 123: 518-527. [Crossref]

3. Chen ZY, Yan MX, Xiang BK, Zhan HW (2001) Alterations of gut hormones of blood and colonic mucosa in rats with chronic stress. Shijie Huaren Xiaohua Zazhi 9: 59-61.

4. Tawata M, Kurihara A, Nitta K, Iwase E, Gan N, et al. (1994) The effects of goshajinkigan, a herbal medicine, on subjective symptoms and vibratory threshold in patients with diabetic neuropathy. Diabetes Res Clin Pract 26: 121-128. [Crossref]

5. Kono T, Mishima H, Shimada M, Morita S, Sakamoto J; GONE Investigators (2009) Preventive effect of goshajinkigan on peripheral neurotoxicity of FOLFOX therapy: a placebo-controlled double-blind randomized phase II study (the GONE Study). Jpn J Clin Oncol 39: 847-849. [Crossref]

6. Honerjäger P, Meissner A (1983) The positive inotropic effect of aconitine. Naunyn Schmiedebergs Arch Pharmacol 322: 49-58. [Crossref] 
7. Nagano T, Itoh H, Takeyama M (1999) Effect of Dai-kenchu-to on levels of 3 braingut peptides (motilin, gastrin and somatostatin) in human plasma. Biol Pharm Bull 22: 1131-1133. [Crossref]

8. Sato Y, Katagiri F, Inoue S, Itoh H, Takeyama M (2004) Dai-kenchu-to raises levels of calcitonin gene-related peptide and substance $\mathrm{P}$ in human plasma. Biol Pharm Bull 27: 1875-1877. [Crossref]

9. Sato Y, Itoh H, Takeyama M (2010) Daikenchuto raises plasma levels of motilin in cancer patients with morphine-induced constipation. J Trad Med 26: 3-5.

10. Sato Y, Hiroki I, Suzuki Y, Tatsuta R, Takeyama M (2013) Interaction between Pirenzepine and Ninjinto, a Traditional Japanese Herbal Medicine, on the Plasma GutRegulated Peptide Levels in Humans. Int J Pept 2013: 5-8. [Crossref]

11. Satoh K, Kase Y, Hayakawa T, Murata P, Ishige A, et al. (2001) Dai-kenchu-to enhances accelerated small intestinal movement. Biol Pharm Bull 24: 1122-1126. [Crossref]

12. Nagano T, Ikawa K, Takeyama M (1998) Enzyme immunoassay of calcitonin generelated peptide-like immunoreactive substance in human plasma and saliva. Jpn J Hosp Pharm 24: 363-369.

13. Takeyama M, Mori K, Takayama F, Kondo K, Kitagawa K, et al. (1990) Enzyme immunoassay of a substance P-like immunoreactive substance in human plasma and saliva. Chem Pharm Bull (Tokyo) 38: 3494-3496. [Crossref]

14. Takeyama M, Wakayama K, Takayama F, Kondo K, Fujii N, et al. (1990) Microenzyme immunoassay of vasoactive intestinal polypeptide (VIP)-like immunoreactive substance in bovine milk. Chem Pharm Bull (Tokyo) 38: 960-962. [Crossref]

15. Takeyama M, Kondo K, Hayashi Y, Yajima H (1989) Enzyme immunoassay of gastrin releasing peptide (GRP)-like immunoreactivity in milk. Int J Pept Protein Res 34: 7074. [Crossref]

16. Kitagawa T, Shimozono T, Aikawa T, Yoshida T, Nishimura H (1981) Preparation and characterization of hetero-bifunctional cross-linking reagents for protein modifications. Chem Pharm Bull 29: 1130-1135.
17. Taché Y, Pappas T, Lauffenburger M, Goto Y, Walsh JH, et al. (1984) Calcitonin generelated peptide: potent peripheral inhibitor of gastric acid secretion in rats and dogs. Gastroenterology 87: 344-349. [Crossref]

18. Sternini C, De Giorgio R, Furness JB (1992) Calcitonin gene-related peptide neurons innervating the canine digestive system. Regul Pept 42: 15-26. [Crossref]

19. Holzer P (1998) Neural emergency system in the stomach. Gastroenterology 114: 823 839. [Crossref]

20. Grider JR (1994) CGRP as a transmitter in the sensory pathway mediating peristaltic reflex. Am J Physiol 266: G1139-1145. [Crossref]

21. Nakamura T, Naruse S, Ozaki T, Kumada K (1996) Calcitonin gene-related peptide is a potent intestinal, but not gastric, vasodilator in conscious dogs. Regul Pept 65 : 211-217. [Crossref]

22. Mizuno K, Kono T, Suzuki Y, Miyagi C, Omiya Y, et al. (2014) Goshajinkigan, a traditional Japanese medicine, prevents oxaliplatin-induced acute peripheral neuropathy by suppressing functional alteration of TRP channels in rat. $J$ Pharmcol Sci 125: 91-98. [Crossref]

23. Ekblad E, Sundler F (1997) Distinct receptors mediate pituitary adenylate cyclaseacitivating peptide-and vasoactive intestinal peptide-induced relaxation of rat ileal longitudinal muscle. Eur J Pharm 334: 61-67. [Crossref]

24. Mitamura M, Horie S, Sakaguchi M, Someya A, Tsuchiya S, et al. (2014) Goshajinkigan, a traditional Japanese medicine, prevents oxaliplatin-induced acute peripheral neuropathy by suppressing functional alteration of TRP channels in rat. $J$ Pharmacol Sci 125: 91-98. [Crossref]

25. Suzuki Y, Goto K, Ishige A, Komatsu Y, Kamei J (1999) Antinociceptive effect of Gosha-jinki-gan, a Kampo medicine, in streptozotocin-induced diabetic mice. Jpn J Pharmacol 79: 169-175. [Crossref]

26. Kummer W, Fischer A, Mundel P, Mayer B, Hoba B, et al. (1992) Nitric oxide synthase in VIP-containing vasodilator nerve fibres in the guinea-pig. Neuroreport 3: 653-655. [Crossref]

Copyright: (C2015 Sato Y. This is an open-access article distributed under the terms of the Creative Commons Attribution License, which permits unrestricted use, distribution, and reproduction in any medium, provided the original author and source are credited. 